\title{
Deregulation of IncRNA HIST1H2AG-6 and AIM1-3 in peripheral blood mononuclear cells is associated with newly diagnosed type 2 diabetes
}

Hui Jiang ${ }^{1 \dagger}$, Peian Lou ${ }^{2+}$, Xiaoluo Chen ${ }^{1}$, Chenguang $\mathrm{Wu}^{1}$ and Shihe Shao ${ }^{3^{*}}$

\begin{abstract}
Background: Type 2 diabetes mellitus (T2DM) is mainly affected by genetic and environmental factors; however, the correlation of long noncoding RNAs (IncRNAs) with T2DM remains largely unknown.

Methods: Microarray analysis was performed to identify the differentially expressed IncRNAs and messenger RNAs (mRNAs) in patients with T2DM and healthy controls, and the expression of two candidate IncRNAs (Inc-HIST1H2AG-6 and Inc-AIM1-3) were further validated using quantitative real-time polymerase chain reaction (qRT-PCR). Spearman's rank correlation coefficient was used to measure the degree of association between the two candidate IncRNAs and differentially expressed mRNAs. Furthermore, the KEGG (Kyoto Encyclopedia of Genes and Genomes) pathway and GO (Gene Ontology) enrichment analysis were used to reveal the biological functions of the two candidate IncRNAs. Additionally, multivariate logistic regression analysis and receiver operating characteristic (ROC) curve analysis were performed.

Results: The microarray analysis revealed that there were 55 IncRNAs and 36 mRNAs differentially expressed in patients with T2DM compared with healthy controls. Notably, Inc-HIST1H2AG-6 was significantly upregulated and IncAIM1-3 was significantly downregulated in patients with T2DM, which was validated in a large-scale qRT-PCR examination (90 controls and 100 patients with T2DM). Spearman's rank correlation coefficient revealed that both IncRNAs were correlated with 36 differentially expressed mRNAs. Furthermore, functional enrichment (KEGG and GO) analysis demonstrated that the two IncRNA-related mRNAs might be involved in multiple biological functions, including cell programmed death, negative regulation of insulin receptor signal, and starch and sucrose metabolism. Multivariate logistic regression analysis revealed that Inc-HIST1H2AG-6 and Inc-AIM1-3 were significantly correlated with T2DM $(\mathrm{OR}=5.791$ and 0.071 , respectively, both $P=0.000)$. Furthermore, the ROC curve showed that the expression of IncHIST1H2AG-6 and Inc-AIM1-3 might be used to differentiate patients with T2DM from healthy controls (area under the ROC curve $=0.664$ and 0.769 , respectively).
\end{abstract}

Conclusion: The profiles of IncRNA and mRNA were significantly changed in patients with T2DM. The expression levels of Inc-HIST1H2AG-6 and Inc-AIM1-3 genes were significantly correlated with some features of T2DM, which

\footnotetext{
*Correspondence: shaoshihe2006@163.com

${ }^{\dagger}$ Hui Jiang and Peian Lou are co-first authors and contributed equally

${ }^{3}$ School of Medicine, Jiangsu University, 301 Xuefu Road,

Zhenjiang 212013, Jiangsu Province, China

Full list of author information is available at the end of the article
}

(c) The Author(s) 2021. This article is licensed under a Creative Commons Attribution 4.0 International License, which permits use, sharing, adaptation, distribution and reproduction in any medium or format, as long as you give appropriate credit to the original author(s) and the source, provide a link to the Creative Commons licence, and indicate if changes were made. The images or other third party material in this article are included in the article's Creative Commons licence, unless indicated otherwise in a credit line to the material. If material is not included in the article's Creative Commons licence and your intended use is not permitted by statutory regulation or exceeds the permitted use, you will need to obtain permission directly from the copyright holder. To view a copy of this licence, visit http://creativecommons.org/licenses/by/4.0/. The Creative Commons Public Domain Dedication waiver (http://creativecommons.org/publicdomain/zero/1.0/) applies to the data made available in this article, unless otherwise stated in a credit line to the data. 
may be used to distinguish patients with T2DM from healthy controls and may serve as potential novel biomarkers for diagnosis in the future.

Keywords: Long noncoding RNA, Type 2 diabetes mellitus, Gene expression, Microarray analysis, Lnc-HIST1H2AG-6, Lnc-AIM1-3

\section{Background}

Diabetes mellitus (DM), one of the most common chronic diseases worldwide, is characterized by elevated blood glucose levels. According to a report by the International Diabetes Federation (IDF), the prevalence of DM in the global population aged 20-79 years is expected to rise from $8.8 \%$ in 2015 to $10.4 \%$ in 2040 . By 2040, the number of patients with DM in the world will increase by $54.7 \%$ [1]. China has the world's largest diabetes epidemic; one study estimated that among adults in China, the overall prevalence of diabetes was $10.9 \%$ and that of prediabetes was $35.7 \%$ in 2013 [2]. Type 2 DM (T2DM) reportedly accounts for $>90 \%$ of all patients with DM [3]. T2DM has become an important public health problem that has serious effects on quality of life due to chronic complications, including nephropathies, retinopathies, neuropathies, and cardiovascular diseases [4]. Therefore, exploring its occurrence and developmental mechanism is important for early diagnosis and treatment of T2DM. In previous decades, noncoding RNAs (ncRNAs), involving microRNAs, small interfering RNAs (siRNAs), long noncoding RNAs (lncRNAs), circular RNAs (circRNAs), and piwi-interacting RNAs (piRNAs) have been the focus of research because of their important biological functions that are strongly associated with the occurrence and development of many diseases, including T2DM [5, 6].

LncRNAs are defined as a kind of functional RNA molecule (larger than 200 nucleotides) without the ability of protein coding, but with the functions of chromosome modification, transcriptional regulation, and post-transcriptional processing [7]. LncRNAs appear to be the main regulators of gene expression and govern diverse biological processes of genomic imprinting, metabolism, proliferation, cell differentiation, immune regulation, and apoptosis $[8,9]$. Several studies have focused on the relationship between lncRNAs and various diseases, including diabetes, cardiovascular diseases, neurological diseases, and cancer [10-14]. The abnormal expression of lncRNAs in T2DM has recently been shown in the analysis of the human $\beta$-cell transcriptome; lncRNAs can regulate the secretion of insulin by the islet $\beta$ cells and might play a role in the regulation of glucose metabolism by the liver and skeletal muscles. Maternally expressed gene 3 (MEG3), taurine upregulated gene 1 (TUG1), and the growth inhibition of specific transcription (GAS5) regulate islet $\beta$ cells in the synthesis and secretion of insulin through PDX1 and MafA [15-17]. Zhang et al. found that lncRNA H19 in the liver of mice on a highfat diet was increased and liver-specific overexpression of H19 promoted the production of liver glucose, causing hyperglycemia and insulin resistance (IR), whereas the systemic knockout of H19 increased the inhibitory effect of insulin on hepatic gluconeogenesis [18]. Brown fat lncRNA1 (Blnc1) could promote the differentiation of brown fat. Furthermore, the inhibited expression of Blnc1 in adipose tissue aggravates obesity-related brown fat whiteness and adipose tissue inflammation, leading to more severe IR [19].

Several studies have shown that lncRNAs were closely related to diabetes; however, the abnormal expression and function of lncRNAs in T2DM remain largely unknown [15-19]. Thus, the purpose of this study was to investigate the association of novel lncRNAs with T2DM and to determine potential biomarkers by comprehensive analysis, including microarray analysis (lncRNA and mRNA expression profiles), large-scale validation, multivariate logistic regression analysis, and ROC curve analysis.

\section{Methods \\ Ethics and subjects}

The study was conducted within the Chinese Han population in Jiangsu Province, China, and approved by the Institutional Ethics Committee of the Affiliated People's Hospital of the Jiangsu University, Zhenjiang, Jiangsu, China (K-20170109-Y). Informed consent was provided by all the participants. One-hundred newly diagnosed patients with T2DM (45 men and 55 women; mean age: 49.2 years, range: $18-70$ years) were enrolled from the endocrinology clinic at the Affiliated People's Hospital of the Jiangsu University (Zhenjiang, Jiangsu, China) from December 2017 to May 2018. For the pupose of this study, the diagnosis of T2DM was established according to the World Health Organization (WHO) diabetes diagnostic criteria published in 1999 [20], which defined diabetes based on a fasting plasma glucose concentration of $7.0 \mathrm{mmol} / \mathrm{L}(126 \mathrm{mg} / \mathrm{dL})$ or higher, a 2-h postload plasma glucose concentration of $11.1 \mathrm{mmol} / \mathrm{L}(200 \mathrm{mg} /$ $\mathrm{dL}$ ) or higher, or a random plasma glucose concentration of $11.1 \mathrm{mmol} / \mathrm{L}(200 \mathrm{mg} / \mathrm{dL})$ or higher. Ninety healthy controls (43 men and 47 women; mean age: 46.5 years, 
range: 18-70 years) were recruited from the medical examination center. None of the participants had been treated with any hypoglycemic agent. Those with a history of type 1 diabetes, other special-type diabetes, cancer, pregnancy, immunodeficiency, chronic organ disease, infectious disease, or chronic or acute complications of diabetes were excluded.

\section{Anthropometric and biochemical measurements}

For all participants, blood samples were collected from the cubital vein after an overnight fast of at least $10 \mathrm{~h}$. Body mass index (BMI) was calculated as weight $(\mathrm{kg})$ divided by the square of the height $(\mathrm{m})$. Blood pressure was measured in a calm state.

Total cholesterol (TC), triglyceride, high-density lipoprotein cholesterol (HDL-C), low-density lipoprotein cholesterol (LDL-C), blood glucose, and uric acid (UA) levels were measured using AU5800 Clinical Chemistry System (Beckman Coulter AU5800, Brea, CA, USA). The white blood cell (WBC) count was assessed using an automatic blood cell analyzer (Beckman DxH 500, Brea, CA, USA). The human insulin levels were measured using Access Immunoassay System (Beckman Coulter Unicel DxI 800, Brea, CA, USA). The glycosylated hemoglobin A1c (HbA1C) level was tested using HLC-723G8 (Tosoh Corporation, Tokyo, Japan). Homeostatic model assessment-IR (HOMA-IR) was determined according to the following formula: [fasting plasma glucose $(\mathrm{mmol} / \mathrm{L}) \times$ fasting insulin $(\mathrm{lU} / \mathrm{mL})] / 22.5$. Further, homeostatic model assessment of $\beta$-cell function (HOMA-B) was determined as follows: $[20 \times$ insulin $(\mathrm{lU} / \mathrm{mL}) /($ fasting plasma glucose $(\mathrm{mmol} / \mathrm{L})-3.5] \%$.

\section{Microarray analysis}

Total RNA was extracted from the peripheral blood mononuclear cells (PBMCs) of patients with and without diabetes using the MiniBEST Universal RNA Extraction Kit (TaKaRa, Tokyo, Japan), as per the manufacturer's instructions. RNA integrity was assessed using Agilent Bioanalyzer 2100 (Agilent Technologies, Santa Clara, CA, USA), and the purity was measured using a NanoDrop2000 spectrophotometer (Thermo Scientific, MA, USA). The satisfactory RNA samples [OD260/280 > 1.8, OD260/230 $>1.5$, and RNA Integrity Number $(\mathrm{RIN}) \geq 8.0$ ] were taken for further microarray analysis. Finally, RNA samples from two patients with T2DM and two healthy controls were selected for chip analysis (OEBiotech, Shanghai, China). Sample labeling (labeled with cy3), microarray hybridization, and washing were performed as per the manufacturer's standard protocols. Typically, total RNA was transcribed to double-strand complementary DNA (cDNA) and then synthesized into complementary RNA (cRNA). After that, second-cycle
cDNA was synthesized from cRNA, followed by fragmentation and biotin labeling. The second-cycle cDNA was hybridized onto the microarray. After washing and staining, the arrays were scanned using an Affymetrix Scanner 3000 (Affymetrix, Santa Clara, CA, USA).

\section{Identification of differentially expressed genes}

Affymetrix Gene Chip Command Console Software (version 4.0, Affymetrix) was used to extract the raw data signals. Expression Console Software (version 1.3.1, Affymetrix) was used to offer log scale robust multiarray analysis normalization for both gene and exon level analysis. GeneSrping Software (version 13.1; Agilent Technologies) was used to perform normalization and subsequent data processing. Differentially expressed genes (DEGs) were identified as significantly changed with the threshold of a fold-change (FC) of $\geq 2.0$ (up or down) and a $P$-value of $\leq 0.05$.

\section{Quantitative real-time polymerase chain reaction analysis} PBMC samples from each subject (90 healthy controls and 100 patients with T2DM) were separated from the whole blood using a red cell lysis buffer. Trizol reagent (Invitrogen, Carlsbad, CA, USA) was used to separate the total RNA. After separation, $10 \mathrm{mM}$ of dNTPs, $2 \mu \mathrm{g}$ of total RNA from each sample, $10 \mu \mathrm{M}$ of random hexamers, $80 \mathrm{U}$ of RNase inhibitor, and $200 \mathrm{U}$ of MMLV reverse transcriptase (MBI Fermentas, Hanover, USA) were used and reverse transcribed into single-stranded cDNA; the samples were then stored at $-20^{\circ} \mathrm{C}$.

Two candidate lncRNAs (lnc-HIST1H2AG-6, lnc-AIM1-3) were quantified using quantitative real-time polymerase chain reaction (qRT-PCR) because of their differential expression $(P<0.05)$ (Table 1). Primer sequences used in this study were synthesized by Shanghai Sunny Biotechnology Co., Ltd. (Shanghai, China) and appended as follows: lnc-HIST1H2AG-6: Forward 5'-AGAAGTGAG ATGTAACCAGAG-3', Reverse 5'-ACTGTCTTT GAGGAACTGAC-3'; lnc-AIM1-3: Forward $5^{\prime}-\mathrm{T}$ GCCATAAG AT TCTAACC TCTGC-3', Reverse $5^{\prime}$ -GGG TAA GTG GAA AT T C T T TGTCC-3'; GAP DH: Forward 5'-AGGTGAAGGTCGGAGTCAAC-3', Reverse 5'-GGGTGGAATCATATTGGAACA-3'. The

Table 1 Differential expression of selected IncRNAs identified using microarray analysis in PBMC between T2DM and controls

\begin{tabular}{|c|c|c|c|c|c|}
\hline GeneSymbol & $\begin{array}{l}P \\
\text { value }\end{array}$ & $\begin{array}{l}\text { FC } \\
\text { (abs) }\end{array}$ & Regulation & NonCodelD & Chr \\
\hline $\begin{array}{l}\text { Inc- } \\
\qquad \text { HIST1H2AG-6 }\end{array}$ & 0.0057 & 4.754367 & Up & NONHSAT108316 & chr6 \\
\hline Inc-AIM1-3 & 0.0228 & 2.151183 & Down & NONHSAT114227 & chr6 \\
\hline
\end{tabular}


reverse transcription was performed using $2 \times$ SYBR Green mix (TransGen Biotech, China), and the qRTPCR was performed using the ABI 7500 Fast RT-PCR System (Applied Biosystems, California, USA), the cycle was set at $95{ }^{\circ} \mathrm{C}$ for $5 \mathrm{~min}$, followed by 40 cycles at $95{ }^{\circ} \mathrm{C}$ for $30 \mathrm{~s}, 58{ }^{\circ} \mathrm{C}$ for $30 \mathrm{~s}, 72{ }^{\circ} \mathrm{C}$ for $32 \mathrm{~s}$, and $75{ }^{\circ} \mathrm{C}$ for $32 \mathrm{~s}$ to collect fluorescence. The abundance of each lncRNA transcript was calculated by analyzing the expression of housekeeper gene glyceraldehyde-3-phosphate dehydrogenase (GAPDH) using $2^{-\Delta \Delta C T}$ method [21].

\section{Biological function analysis of Inc-HIST1H2AG-6 and Inc-AIM1-3}

To better understand the biological function of lncHIST1H2AG-6 and lnc-AIM1-3, Spearman's rank correlation test was performed first to screen their degree of association with differentially expressed mRNAs and construct an interaction network by Cytoscape software (version 3.7.0). Furthermore, KEGG Pathway and GO enrichment analysis were performed using the $\mathrm{R}$ software clusterProfiler package to explore the potentially enriched pathway and biological processes of the two lncRNAs related to mRNAs.

\section{Statistical analyses}

Categorical data (including sex) were presented as numbers and percentages. Other data were presented as mean \pm standard deviation (SD) values or medians with interquartile ranges. The normality of distributions was assessed for the variables. Differences between groups were evaluated using the unpaired Student's t-test for data with normal distribution and the Mann-Whitney $U$ test for data with abnormal distribution; $\chi^{2}$ test was used for categorical variables. Multivariate logistic regression analysis was used to analyze the association of T2DM with different variables, including age, BMI, systolic blood pressure (SBP), LDL-C, HDL-C, lncHIST1H2AG-6, and lnc-AIM1-3. The diagnostic value of IncRNAs was evaluated using the receiver operating characteristic (ROC) curve and area under the ROC curve (AUC). SPSS version 20.0 software was used for statistical analysis. $P$-values were two-sided, and $P$-values of $<0.05$ were considered significant. The correlations of lnc-HIST1H2AG-6 and lnc-AIM1-3 with glucose metabolism indexes were assessed using Pearson correlation test.

\section{Results}

\section{Main characteristics of the study cohort}

A total of 190 subjects, including 90 controls and 100 patients with T2DM, were recruited in the present study. The main clinical characteristics of patients with and
Table 2 General characteristic of T2DM and control group for validation study

\begin{tabular}{lllr}
\hline Clinical characteristics & T2DM $(\mathbf{n}=\mathbf{1 0 0})$ & control $(\mathbf{n}=\mathbf{9 0})$ & P value \\
\hline Sex (male/female) & $45 / 55$ & $43 / 47$ & 0.701 \\
Age (years) & $49.2 \pm 12.2$ & $46.5 \pm 10.4$ & 0.302 \\
BMI (kg/m²) & $24.2(22.9-25.9)$ & $20.8(20.1-24.5)$ & 0.047 \\
DBP $(\mathrm{mmHg})$ & $78(70-80)$ & $73(68-80)$ & 0.124 \\
SBP $(\mathrm{mmHg})$ & $138(130-140)$ & $130(125-140)$ & 0.003 \\
WBC $\left(\times 10^{9} / \mathrm{L}\right)$ & $4.8(4.4-5.7)$ & $4.7(4.5-5.8)$ & 0.984 \\
FPG $(\mathrm{mmol} / \mathrm{L})$ & $10.7(8.8-13.2)$ & $5.3(4.9-5.8)$ & 0.000 \\
HbA1c $(\%)$ & $9.3(8.0-11.5)$ & $4.8(4.4-5.1)$ & 0.000 \\
FINS (ulu/ml) & $5.3(3.7-8.3)$ & $7.2(6.7-8.7)$ & 0.000 \\
HOMA-B & $11.7(6.5-24)$ & $108.8(91.6-141.9)$ & $<0.001$ \\
HOMA-IR & $2.5(1.6-4.1)$ & $1.6(1.4-2.0)$ & $<0.001$ \\
TG (mmol/l) & $1.7(1.0-2.3)$ & $1.2(0.9-1.8)$ & 0.278 \\
LDL-C (mmol/l) & $2.8 \pm 0.8$ & $2.5 \pm 0.5$ & 0.002 \\
HDL-C (mmol/l) & $1.1 \pm 0.3$ & $1.3 \pm 0.3$ & 0.000 \\
UA (mmol/L) & $336.1 \pm 59.5$ & $330.5 \pm 79.5$ & 0.576 \\
\hline
\end{tabular}

Data are expressed as mean \pm standard deviation values, numbers, or medians (interquartile ranges). Data were analyzed using the unpaired Student's t-test, $\mathrm{x}^{2}$-test and Mann-Whitney test, as appropriate

$\mathrm{BMI}$, body mass index; DBP, diastolic blood pressure; SBP, systolic blood pressure; WBC, white blood cell; FPG, fasting plasma glucose; $\mathrm{HbA1c}$, haemoglobin A1c; FIN, fasting insulin; TG, triglyceride; LDL-C, low density lipoprotein cholesterol; $\mathrm{HDL}-\mathrm{C}$, high density lipoprotein cholesterol; UA, urine acid

without diabetes are presented in Table 2. There were no significant differences in the distribution of sex, age, diastolic blood pressure (DBP), WBC, triglyceride, and UA between patients with T2DM and healthy controls $(P>0.05)$. However, the BMI, SBP, fasting plasma glucose (FPG), HbA1c, fasting insulin (FIN), HOMA-IR, HOMA-B, LDL-C, and HDL-C of patients with T2DM were significantly different from those of healthy controls $(P<0.05$ or $P<0.01)$, indicating that these blood samples from the subjects described above could be used for the following experimental analysis.

\section{Expression profile of IncRNA and mRNA by microarray analysis}

In the present study, we compared the gene expression profile displayed by PBMCs from patients with T2DM with that of healthy controls by using the GeneSpring Software. The microarray cohort was composed of two patients who were newly diagnosed with T2DM and two healthy controls. There were 55 lncRNAs differently expressed in patients with T2DM compared with that in healthy controls; the detailed information was listed in Additional file 1. Among these 55 lncRNAs, 18 were upregulated and 37 were downregulated. In addition, we also identified 36 mRNAs that had aberrant expression between patients with T2DM and healthy controls; 
among these, 16 mRNAs were upregulated, and 20 mRNAs were downregulated. The detailed information about these 36 differently expressed mRNAs is presented in Additional file 2.

\section{Validation of two candidate IncRNAs}

Two candidate lncRNAs (lnc-HIST1H2AG-6 and lncAIM1-3) were chosen from the differentially expressed lncRNAs $\quad(\mathrm{FC} \geq 2.0$ or $\leq-2.0, \quad P<0.05)$ for further large-scale validation (90 controls and 100 patients with T2DM). QRT-PCR analysis of the blood samples of T2DM and healthy controls was performed. As shown in Fig. 1, compared with that in healthy controls, the HIST1H2AG-6 transcript level in patients with T2DM was significantly upregulated $(P=0.01)$, whereas the expression of AIM1-3 was significantly downregulated $(P<0.01)$, which were consistent with the results of microarray analysis, indicating the reliability of our data and the potential role of two candidate lncRNAs in T2DM.

\section{Biological function analysis of the two candidate IncRNAs}

To better understand the biological functions of the two candidate lncRNAs (lnc-HIST1H2AG-6 and lncAIM1-3) and their potential role in T2DM, Spearman's rank correlation and functional enrichment analysis were performed. Spearman's rank correlation revealed that the expression levels of lnc-HIST1H2AG-6 and lncAIM1-3 were correlated with 36 differentially expressed mRNAs (Fig. 2). Furthermore, lnc-HIST1H2AG-6 and lnc-AIM1-3 related mRNAs were enriched in multiple
KEGG pathways (eg, starch and sucrose metabolism, NOD-like receptor signaling pathway, metabolic pathways, and MAPK signaling pathway) and GO terms (eg, cellular metabolic process, immune response and signal transduction). The top 30 KEGG pathway and GO terms are presented in Fig. 3, and the detailed information of KEGG pathway and GO terms is listed in Table 3 and Additional file 3 , respectively. The enriched pathways (eg, starch and sucrose metabolism and MAPK signaling pathway) and related genes (eg, IL1B and ENPP1) have been reported association with T2DM [22-24], which indicated that lnc-HIST1H2AG-6 and lnc-AIM1-3 might play a role in T2DM.

\section{Correlations between the two IncRNAs expression and parameters in T2DM}

Correlation analysis was used to investigate whether lnc-HIST1H2AG-6 and lnc-AIM1-3 were associated with clinical and laboratory features in our cohort of T2DM. According to our study, lnc-HIST1H2AG-6 was positively correlated with LDL-C and UA $(P=0.026$ and $P=0.021$ respectively) and negatively correlated with HOMA-B $(P=0.039)$; lnc-AIM1-3 had a positive correlation with FIN, HOMA-B, and HDL-C $(P=0.003$, $P<0.001$, and $P<0.001$, respectively) and a negative correlation with FPG and HbA1c $(P<0.001$ and $P<0.001$, respectively) (Table 4).

\section{Multivariate logistic regression}

Using multivariate logistic regression (Table 5), we observed five variables (BMI, LDL-C, HDL-C,
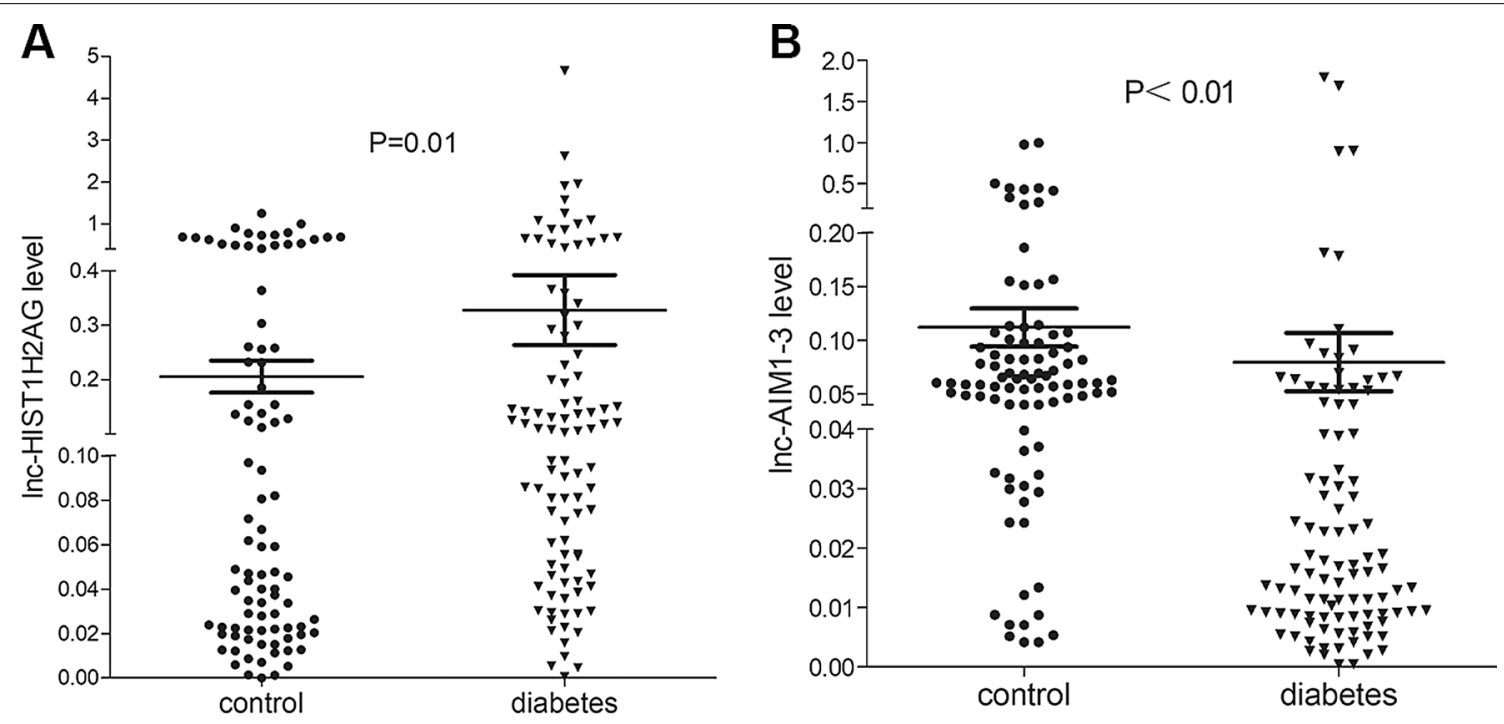

Fig. 1 Relative expression levels of Inc-HIST1H2AG-6 (A) and Inc-AIM1-3 (B) in patients with T2DM and controls. The transcript levels were evaluated using qRT-PCR 

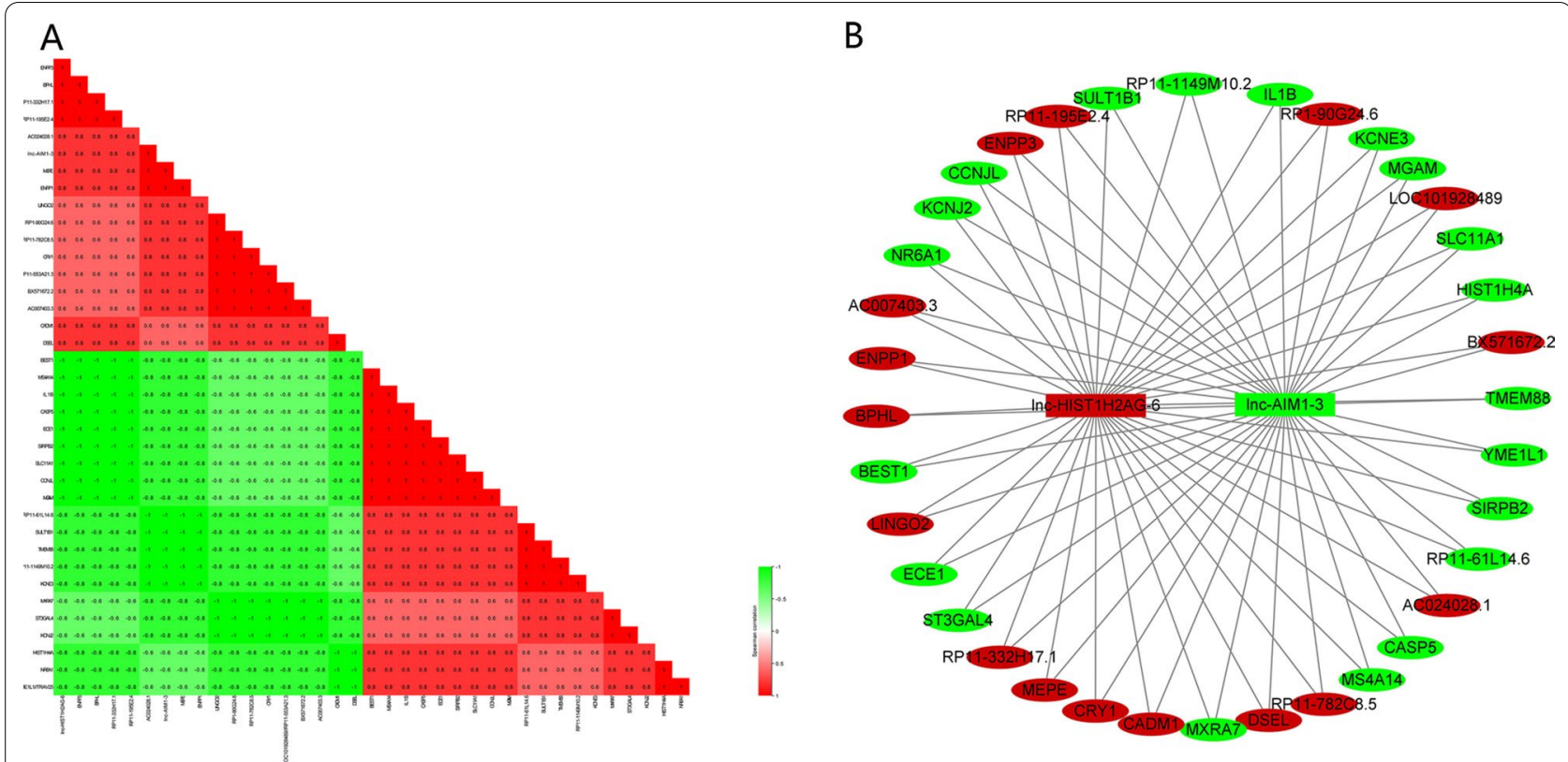

Fig. 2 Correlation of Inc-HIST1H2AG-6 and Inc-AIM1-3 with differential expression mRNAs. (A) Spearman rank coefficient, red color indicates positive correlation and green color indicates negative correlation (B) Interaction network, red color indicates upregulated and green color indicates downregulated in T2DM

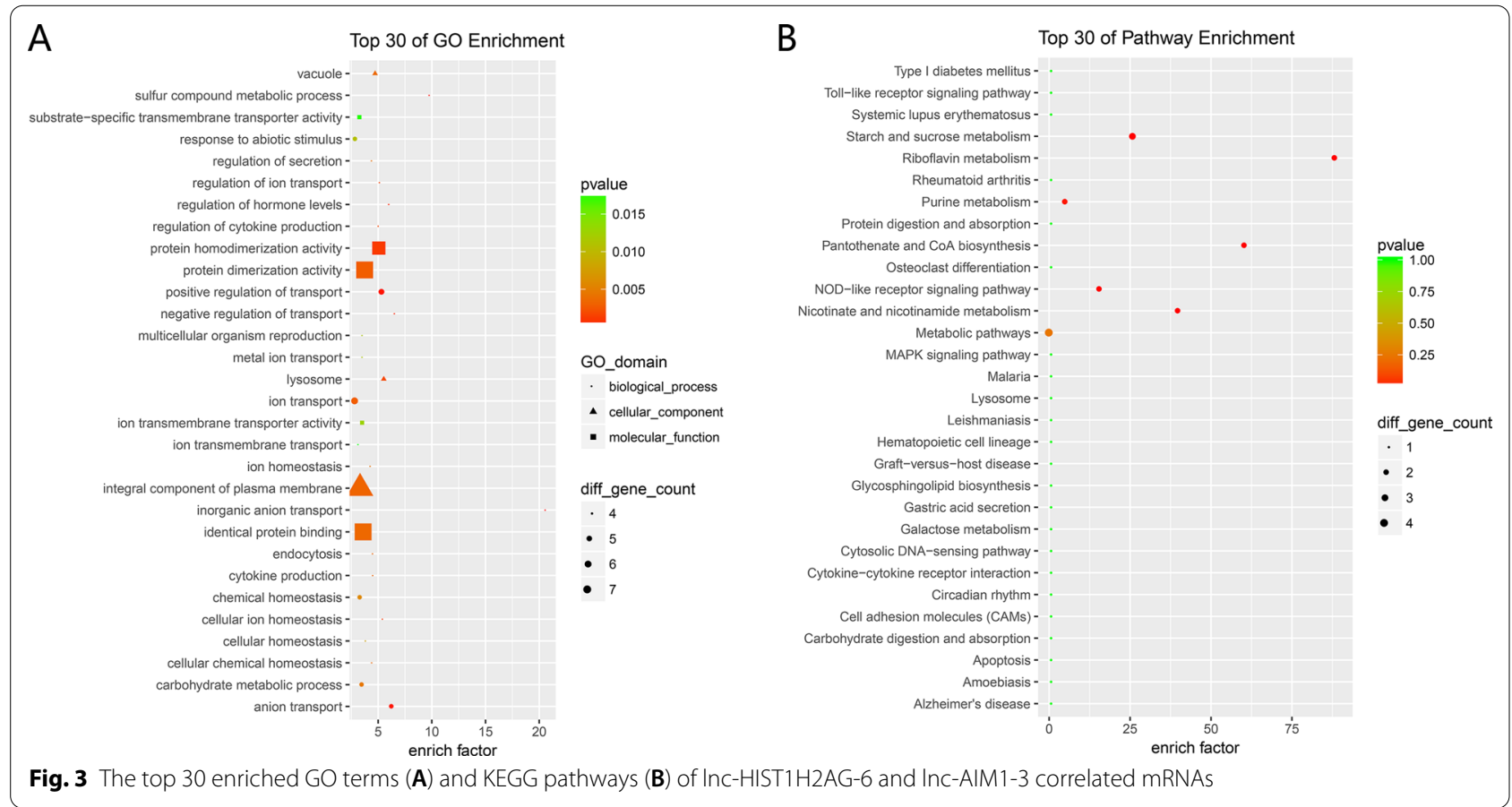

lnc-HIST1H2AG-6, and lnc-AIM1-3) that were significantly correlated with T2DM. For example, the expression of lnc-AIM1-3 was negatively correlated with T2DM $[\beta=-2.54$, odds ratio $(\mathrm{OR})=0.071,95 \%$ confidence interval $(\mathrm{CI})=0.029-0.178, \quad P=0.000], \quad$ and lncHIST1H2AG-6 expression was positively associated with T2DM $(\beta=1.756, O R=5.791,95 \% C I=2.275-14.739$, 
Table 3 Enriched pathways of Inc-HIST1H2AG-6N and Inc-AIM1-335 related mRNAs

\begin{tabular}{|c|c|c|}
\hline KEGG pathway & Genes count & Enriched genes \\
\hline Riboflavin metabolism & 2 & ENPP3 ENPP1 \\
\hline Pantothenate and $\mathrm{CoA}$ biosynthesis & 2 & ENPP3 ENPP1 \\
\hline Nicotinate and nicotinamide metabolism & 2 & ENPP3 ENPP1 \\
\hline Starch and sucrose metabolism & 3 & MGAM ENPP3 ENPP1 \\
\hline NOD-like receptor signaling pathway & 2 & IL1B CASP5 \\
\hline Purine metabolism & 2 & ENPP3 ENPP1 \\
\hline Metabolic pathways & 4 & MGAM ST3GAL4 ENPP3 ENPP1 \\
\hline Osteoclast differentiation & 1 & IL1B \\
\hline Carbohydrate digestion and absorption & 1 & MGAM \\
\hline Lysosome & 1 & SLC11A1 \\
\hline Apoptosis & 1 & IL1B \\
\hline Rheumatoid arthritis & 1 & IL1B \\
\hline Cytokine-cytokine receptor interaction & 1 & IL1B \\
\hline Alzheimer's disease & 1 & IL1B \\
\hline Type I diabetes mellitus & 1 & IL1B \\
\hline Circadian rhythm & 1 & CRY1 \\
\hline Leishmaniasis & 1 & $\mathrm{IL} 1 \mathrm{~B}$ \\
\hline Systemic lupus erythematosus & 1 & HIST1H4A \\
\hline Glycosphingolipid biosynthesis & 1 & ST3GAL4 \\
\hline Graft-versus-host disease & 1 & IL1B \\
\hline Toll-like receptor signaling pathway & 1 & IL1B \\
\hline Gastric acid secretion & 1 & KCNJ2 \\
\hline Amoebiasis & 1 & IL1B \\
\hline Galactose metabolism & 1 & MGAM \\
\hline Hematopoietic cell lineage & 1 & IL1B \\
\hline Cell adhesion molecules (CAMs) & 1 & CADM1 \\
\hline Malaria & 1 & IL1B \\
\hline Cytosolic DNA-sensing pathway & 1 & IL1B \\
\hline MAPK signaling pathway & 1 & IL1B \\
\hline Protein digestion and absorption & 1 & KCNE3 \\
\hline African trypanosomiasis & 1 & IL1B \\
\hline Prion diseases & 1 & IL1B \\
\hline Chagas disease (American trypanosomiasis) & 1 & IL1B \\
\hline
\end{tabular}

$P=0.000)$. The results implicated a potential role of lncHIST1H2AG-6 and lnc-AIM1-3 in T2DM.

\section{Diagnostic potential of Inc-HIST1H2AG-6 and Inc-AIM1-3}

To evaluate whether the two lncRNAs could be used to distinguish patients with T2DM and healthy controls, ROC curve analysis was performed and AUC was calculated for lnc-HIST1H2AG-6 and lnc-AIM1-3. As shown in Fig. 4, the AUC for lnc-HIST1H2AG-6 was $0.664(95 \% \mathrm{CI}=0.549-0.780, P=0.007)$ and that for lnc-AIM1-3 was 0.769 (95\% CI=0.662-0.875, $P<0.001)$. The results indicated that both lncHIST1H2AG-6 and lnc-AIM1-3 might be used to distinguish patients with T2DM and healthy controls.

\section{Discussion}

T2DM is characterized by impaired insulin secretion and IR. The prevalence of T2DM and prediabetes has been increasing rapidly during the previous three decades, especially in developing countries, making it one 
Table 4 Correlations between expressions of the two candidate IncRNAs and metabolic characteristics

\begin{tabular}{|c|c|c|c|c|}
\hline \multirow{2}{*}{$\begin{array}{l}\text { Demographic } \\
\text { and metabolic } \\
\text { characteristics }\end{array}$} & \multicolumn{2}{|c|}{ Inc-HIST1H2AG-6 } & \multicolumn{2}{|c|}{ Inc-AIM1-3 } \\
\hline & $r$ & $\mathbf{P}$ & $r$ & $\mathbf{P}$ \\
\hline Age & 0.007 & 0.945 & -0.380 & -0.734 \\
\hline BMI $\left(\mathrm{kg} / \mathrm{m}^{2}\right)$ & 0.101 & 0.338 & -0.930 & 0.456 \\
\hline SBP (mmHg) & 0.192 & 0.056 & -0.181 & -0.181 \\
\hline $\mathrm{DBP}(\mathrm{mmHg})$ & 0.108 & 0.285 & -0.121 & -0.121 \\
\hline FPG (mmol/L) & 0.197 & 0.060 & -0.493 & -0.493 \\
\hline HbA1c (\%) & 0.172 & 0.101 & -0.477 & -0.477 \\
\hline FIN (ulu/ml) & -0.184 & 0.079 & 0.327 & 0.327 \\
\hline HOMA- $\beta$ & -0.216 & 0.039 & 0.507 & 0.507 \\
\hline HOMA-IR & 0.043 & 0.685 & -0.106 & -0.106 \\
\hline TG $(\mathrm{mmol} / \mathrm{l})$ & 0.197 & 0.060 & 0.021 & 0.021 \\
\hline LDL-C (mmol/l) & 0.274 & 0.026 & -0.078 & -0.078 \\
\hline $\mathrm{HDL}-\mathrm{C}(\mathrm{mmol} / \mathrm{l})$ & 0.050 & 0.639 & 0.394 & 0.394 \\
\hline $\mathrm{UA}(\mathrm{mmol} / \mathrm{L})$ & 0.231 & 0.021 & -0.062 & -0.062 \\
\hline
\end{tabular}

BMI, body mass index; DBP, diastolic blood pressure; SBP, systolic blood pressure; WBC, white blood cell; $F P G$, fasting plasma glucose; $\mathrm{HbA1c}$, haemoglobin A1c; FIN, fasting insulin; TG, triglyceride; LDL-C, low density lipoprotein cholesterol; $\mathrm{HDL}-\mathrm{C}$, high density lipoprotein cholesterol; UA, urine acid

of the most vital public health challenges. At present, the islet tissue samples of "gold samples" are extremely difficult to obtain clinically; therefore, if the circulating lncRNA can be used as a clinical marker, it will be more valuable for human physiological and pathological conditions, including T2DM. Recently, several lncRNAs have been proved to be widely involved in many biological processes, such as cell proliferation, differentiation, cycle regulation, and apoptosis [25-27]. It was reported that circulating lncRNAs are potential diagnostic and prognostic factors in diabetes $[28,29]$. The lncRNAs KCNQ1OT1 and ANRIL have been identified as genetic susceptibility loci associated with T2DM [30, 31]. Imamura, et al. revealed that rs7656416 near CTBP1-AS2 were significantly associated with T2DM
[32]. Willer et al. showed that rs10904908 at the VIMCUBN locus on chromosome 10 was associated with lipid levels [33], indicating that lncRNAs might play an important role in regulating gene expression and could be explored as a specific biomarker for T2DM diagnosis and prediction.

PBMCs are a component of the blood that mainly consists of monocytes or macrophages and lymphocytes. In the human genome, PBMCs show a large proportion (about $80 \%$ ) of the coding genes and are easy to determine as a marker in human diseases [34, 35]. Recent research on PBMC has indicated that the deregulation of some lncRNAs might be associated with T2DM. Mohamadi et al. have revealed that the expression of SNHG17 and TTC28AS1 in PBMCs was downregulated in patients with T2DM compared with that in healthy controls, indicating that the downregulation of the lncRNAs TTC28AS1 and SNHG17 in patients with T2DM was associated with T2DM susceptibility [36]. Similarly, Sathishkumar et al. provided the first preliminary evidence that the expression of the lncRNAs THRIL and SALRNA1 in PBMC was decreased in patients with T2DM and negatively correlated with hyperglycemia, senescence, and inflammation. Omidvar et al. reported that decreased levels of VIM-AS1 and CTBP1-AS2 in PBMCs were associated with diabetes in an Iranian population, implying that the expression levels of IncRNA VIM-AS1 and lncRNA CTBP1-AS2 might be associated with T2DM susceptibility [37]. Thus, we reasoned that DEGs in the PMBCs of T2DM and healthy controls might offer a better understanding of the underlying pathology of T2DM and might provide novel therapeutic targets and biomarkers.

In the present study, microarray analysis was conducted to screen the IncRNA and mRNA expression profiles in PBMCs of newly diagnosed patients with T2DM and healthy controls; 55 lncRNAs (18 upregulated and 37 downregulated) and 36 mRNAs (16 upregulated and 20 downregulated) were differentially expressed in patients with T2DM compared with healthy controls.

Table 5 Multivariate logistic regression analysis to reveal the association of T2DM with different variables including age, BMI, SBP, LDLC, HDL-C, Inc-HIST1H2AG-6 and Inc-AIM1-3

\begin{tabular}{lclllll}
\hline Factors & $\boldsymbol{\beta}$ & SE & Wald & P & OR & 95\% Cl \\
\hline Age (years) & -0.003 & 0.020 & 0.026 & 0.871 & 0.997 & $0.958-1.037$ \\
BMl $\left(\mathrm{kg} / \mathrm{m}^{2}\right)$ & 0.200 & 0.071 & 8.006 & 0.005 & 1.222 & $1.063-1.403$ \\
SBP $(\mathrm{mmHg})$ & 0.026 & 0.019 & 7.081 & 0.176 & 1.026 & $0.988-1.066$ \\
LDL $(\mathrm{mmol} / \mathrm{l})$ & 1.014 & 0.373 & 7.403 & 0.007 & 2.755 & $1.328-5.718$ \\
HDL $(\mathrm{mmol} / \mathrm{l})$ & -3.057 & 0.941 & 13.878 & 0.000 & 0.030 & $0.005-0.190$ \\
IncRNAHIST1H2AG-6 & 1.756 & 0.477 & 13.575 & 0.000 & 5.791 & $2.275-14.739$ \\
IncRNAAIM1-3 & -2.54 & 0.466 & 32.18 & 0.000 & 0.071 & $0.029-0.178$ \\
\hline
\end{tabular}

BMI, body mass index; SBP, systolic blood pressure; LDL-C, low density lipoprotein cholesterol; HDL-C, high density lipoprotein cholesterol 

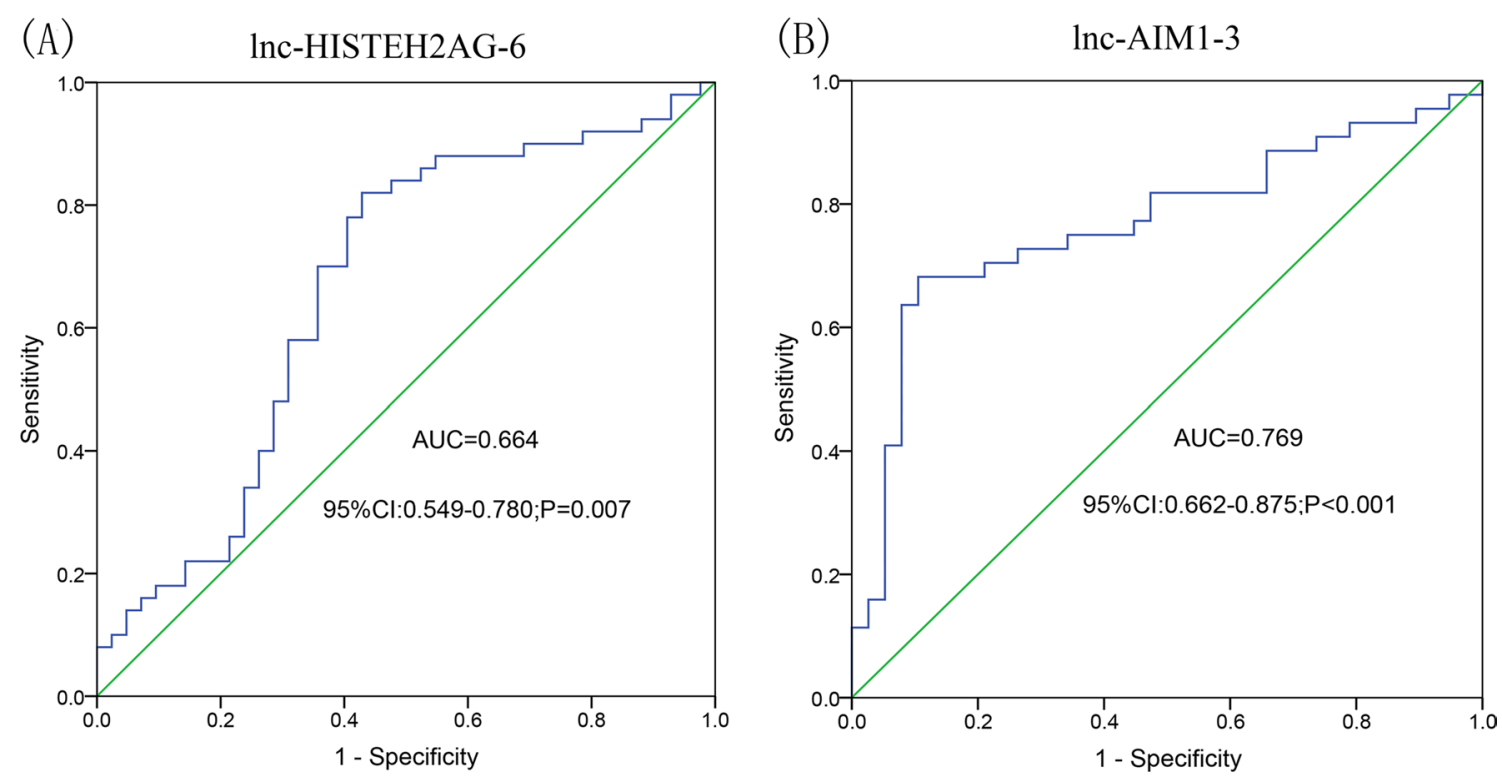

Fig. 4 ROC curves of Inc-HIST1H2AG-6 (A) and Inc-AIM1-3 (B)

The expression levels of two candidate lncRNAs (lncHIST1H2AG-6 and lnc-AIM1-3) were validated by a large-scale qRT-PCR experiment. To better understand the biological function of lnc-HIST1H2AG-6 and lncAIM1-3, their correlated mRNAs were screened and functional enrichment analysis was performed. Our results indicated that lnc-HIST1H2AG-6 and lnc-AIM1-3 were correlated with 36 differentially expressed mRNAs (such as CADM1, IL1B, and ENPP1), which might be involved in many KEGG pathways (such as starch and sucrose metabolism and MAPK signaling pathway). It has been reported that with CADM1, an immunoglobulin superfamily member, ectodomain shedding contributed to blood glucose dysregulation in T2DM [38]. Gene variants or polymorphisms of IL1B and ENP1 have been found to correlate with T2DM in many human studies [39-41]. Moreover, starch and sucrose metabolism and MAPK signaling pathways have been implicated in the development of T2DM in multiple animal models and human studies [42, 43]. The findings of the present study suggested that lnc-HIST1H2AG-6 and lnc-AIM1-3 play an important role in T2DM; thus, further investigation should be performed.

Correlations between the two candidate lncRNAs (lnc-HIST1H2AG-6 and lnc-AIM1-3) and different variables, including FPG, HbA1c, FIN and HOMA-B were identified in our cohort. Our results indicated that lncHIST1H2AG-6 was positively correlated with FPG, triglyceride, LDL-C, and UA and negatively correlated with HOMA-B. However, lnc-AIM1-3 exhibited a positive correlation with FIN, HOMA-B, and HDL-C and a negative correlation with SBP, FPG, and HbA1c. These results were similar to a previous study which reported that the lncRNAs (n342533, n335556, and n336109) were closely correlated to BMI, FPG, 2hPG, HbA1c, HOMAIR, alanine transaminase, HDL-C, and HOMA-B of patients with T2DM and healthy controls [44]. On the basis of these correlations between the two candidate lncRNAs and metabolic characteristics (FPG, HbAlc, LDL-C, HDL-C, FINs, and HOMA-B), we could conclude that lnc-HIST1H2AG-6 and lnc-AIM1-3 might be involved in glucose regulation, lipid homeostasis, and the secretion of insulin. Impaired function of islet $\beta$ cells and reduced insulin sensitivity are the two remarkable characteristics of T2DM. Accumulating evidence suggests that lncRNAs could affect apoptosis and insulin secretion in the pancreatic $\beta$ cells $[15,17,45]$. Recently, increasing evidence has indicated that lncRNAs may act as a link between insulin signaling and IR [46]. Ruan et al. found that the overexpression of IncRNA-p3134 could reduce the apoptosis of islet cells and increase the level of glucose-stimulated insulin by positively regulating the PI3K/ AKT/mTOR (phosphoinositide-3-kinase/protein kinase $\mathrm{B} /$ mammalian target of rapamycin) signaling pathway [47]. As mentioned above, lnc-HIST1H2AG-6 and lncAIM1-3 might be involved in multiple KEGG pathways; further functional studies should be performed to identify their underlying mechanisms in T2DM.

Furthermore, our multivariate logistic regression analysis revealed that some variables (such as BMI, LDL-C, and HDL-C) were significantly correlated with T2DM, which was consistent with previous studies to some 
extent. For example, Mohamadi, et al. showed that the increase in FBG, SBP, and BMI was associated with an increased likelihood of exhibiting T2DM [36]. Pan et al. have reported that elevated LDL-C levels significantly increased the risk of T2DM [48]. Some reports have shown that hypertension was an independent risk factor for T2DM $[49,50]$. However, we observed that the association of SBP with T2DM was not statistically significant. We believed that this could be due to the small sample size of our study or the source of our samples, as lncRNAs displayed tissue-specific patterns of expression [51]. It is worth noting that our results suggested that the expression levels of lnc-HIST1H2AG-6 and lnc-AIM1-3 are significantly correlated with $\mathrm{T} 2 \mathrm{DM}$, which might be used to distinguish patients with T2DM and healthy controls. Further investigation may obtain novel lncRNA biomarkers of T2DM in the future.

Our study has the following limitations: (1) The alterations observed in the PBMCs might be just appearances of the tissue-specific and heterogeneous actions of lncRNAs. (2) This was a single-center study with a relatively small sample size, which needed further large-scale validation in multicenters with comprehensive investigation in the future. (3) With respect to the microarray analysis, there were only two samples in each group, and only two IncRNAs have been verified; the relationship between the other 53 differentially expressed lncRNAs and diabetes warrants further exploration. (4) Based on the present results, it is not possible to infer the causal relationship between these two lncRNAs changes and type 2 diabetes; repeated and prospective follow-up studies are needed. Next, we will further study the role of both lncRNAs in the pathogenesis of diabetes in insulinoma cells and diabetic mice.

\section{Conclusion}

The profiles of lncRNA and mRNA were significantly changed in the patients with T2DM compared with healthy controls. The expression levels of lncHIST1H2AG-6 and lnc-AIM1-3 were significantly correlated with some features of T2DM, which might be used to distinguish patients with T2DM and healthy controls and serve as potential novel biomarkers for diagnosis in the future.

\section{Abbreviations}

T2DM: Type 2 diabetes mellitus; IncRNAs: Long noncoding RNA; qRT-PCR: Quantitative real-time polymerase chain reaction; KEGG: Kyoto Encyclopedia of Genes and Genomes; GO: Gene ontology; ROC: Receiver operating characteristic; DM: Diabetes mellitus; IDF: International Diabetes Federation; ncRNAs: Noncoding RNAs; siRNAs: Small interfering RNAs; circRNA: Circular RNA; piRNA: Piwi-interacting RNA; MEG3: Maternally expressed gene 3;TUG1: Taurine raised gene 1; IR: Insulin resistance; BInc1: Brown fat IncRNA1; WHO: World Health Organization; BMI: Body mass index; TC: Total cholesterol; TG: Triglyceride; HDL-C: High-density lipoprotein cholesterol; LDL-C: Low-density lipoprotein cholesterol; UA: Uric acid; HbA1C: Hemoglobin A1c; HOMA-IR: Homeostasis model assessment-IR; PBMCs: Peripheral blood mononuclear cells; DEGs: Differentially expressed genes; SD: Standard deviation.

\section{Supplementary Information}

The online version contains supplementary material available at https://doi. org/10.1186/s12920-021-00994-z.

Additional file 1. Differentially expressed IncRNAs in patients with T2DM compared with healthy controls.

Additional file 2. Differentially expressed mRNAs in patients with T2DM compared with healthy controls.

Additional file 3. Detailed information of enriched GO terms of IncHIST1H2AG-6 and Inc-AIM1-3 correlated mRNAs.

\section{Acknowledgements}

We thank all of the volunteers for participating in our study. We thank Dr Wen Peng (School of Medicine, Qinghai University) for her linguistic assistance during the preparation of this manuscript.

\section{Authors' contributions}

HJ, CGW and SHS designed the research and wrote the paper. PAL performed the microarray expression profile. $\mathrm{HJ}, \mathrm{XLC}$ were responsible for cases collection and data analysis. All authors read and approved the final manuscript.

\section{Funding}

This work was supported by Postgraduate Research \& Practice Innovation Program of Jiangsu Province (KYCX18_2284) and the social development guide technology plan of Zhenjiang city (FZ2017026). These programs provided financial support for this study.

Availability of data and materials

The datasets generated and/or analysed during the current study are available in the GEO repository, https://www.ncbi.n/m.nih.gov/geo/query/acc.cgi?acc= GSE168437. (Accession number: ezmfqucejvsjfwx).

\section{Declarations}

\section{Ethics approval and consent to participate}

The study was approved by the Institutional Ethics Committee of the Affiliated People's Hospital of the Jiangsu University, Zhenjiang, Jiangsu, China (K-20170109-Y). Written informed consent was provided by all the participants.

\section{Consent for publication}

Not applicable. 


\section{Author details}

${ }^{1}$ Department of Endocrinology, Affiliated People's Hospital of Jiangsu University, Zhenjiang 212002, China. ${ }^{2}$ Xuzhou Center for Disease Control Prevention, Xuzhou 221000, China. ${ }^{3}$ School of Medicine, Jiangsu University, 301 Xuefu Road, Zhenjiang 212013, Jiangsu Province, China.

Received: 29 April 2020 Accepted: 31 May 2021 Published online: 06 June 2021

\section{References}

1. Ogurtsova $K$, da Rocha Fernandes JD, Huang Y, Linnenkamp U, Guariguata L, Cho NH, et al. IDF Diabetes Atlas: global estimates for the prevalence of diabetes for 2015 and 2040. Diabetes Res Clin Pract. 2017;128:40-50

2. Wang L, Gao P, Zhang M, Huang Z, Zhang D, Deng Q, et al. Prevalence and ethnic pattern of diabetes and prediabetes in China in 2013. JAMA. 2017;317(24):2515-23.

3. Khan RMM, Chua ZJY, Tan JC, Yang Y, Liao Z, Zhao Y. From pre-diabetes to diabetes: diagnosis, treatments and translational research. Medicina. 2019;55(9):546.

4. Afghahi H, Miftaraj M, Svensson AM, Hadimeri H, Gudbjornsdottir S, Eliasson $\mathrm{B}$, et al. Ongoing treatment with renin-angiotensin-aldosteroneblocking agents does not predict normoalbuminuric renal impairment in a general type 2 diabetes population. J Diabetes Complicat. 2013;27(3):229-34.

5. Groen JN, Capraro D, Morris KV. The emerging role of pseudogene expressed non-coding RNAs in cellular functions. Int J Biochem Cell Biol. 2014;54:350-5.

6. Della Bella E, Menzel U, Basoli V, Tourbier C, Alini M, Stoddart MJ. Differential regulation of circRNA, miRNA, and piRNA during early osteogenic and chondrogenic differentiation of human mesenchymal stromal cells. Cells. 2020;9(2):398

7. Jia H, Osak M, Bogu GK, Stanton LW, Johnson R, Lipovich L. Genome-wide computational identification and manual annotation of human long noncoding RNA genes. RNA. 2010;16(8):1478-87.

8. Feng SD, Yang JH, Yao CH, Yang SS, Zhu ZM, Wu D, et al. Potential regulatory mechanisms of IncRNA in diabetes and its complications. Biochem Cell Biol. 2017;95(3):361-7.

9. Sun $X$, Wong D. Long non-coding RNA-mediated regulation of glucose homeostasis and diabetes. Am J Cardiovasc Dis. 2016;6(2):17-25.

10. Hu G, Niu F, Humburg BA, Liao K, Bendi S, Callen S, et al. Molecular mechanisms of long noncoding RNAs and their role in disease pathogenesis. Oncotarget. 2018;9(26):18648-63.

11. DiStefano JK. Beyond the protein-coding sequence: noncoding RNAs in the pathogenesis of type 2 diabetes. Rev Diabet Stud. 2015;12(3-4):260-76.

12. Haemmig S, Simion V, Yang D, Deng Y, Feinberg MW. Long noncoding RNAs in cardiovascular disease, diagnosis, and therapy. Curr Opin Cardiol. 2017;32(6):776-83.

13. Renganathan A, Felley-Bosco E. Long noncoding RNAs in cancer and therapeutic potential. Adv Exp Med Biol. 2017;1008:199-222.

14. Floris G, Zhang L, Follesa P, Sun T. Regulatory role of circular RNAs and neurological disorders. Mol Neurobiol. 2017:54(7):5156-65.

15. Wang N, Zhu Y, Xie M, Wang L, Jin F, Li Y, et al. Long noncoding RNA Meg3 regulates mafa expression in mouse beta cells by inactivating Rad21, Smc3 or Sin3alpha. Cell Physiol Biochem. 2018;45(5):2031-43.

16. Jin F, Wang N, Zhu Y, You L, Wang L, De W, et al. Downregulation of long noncoding RNA Gas5 affects cell cycle and insulin secretion in mouse pancreatic beta cells. Cell Physiol Biochem. 2017;43(5):2062-73.

17. Yin DD, Zhang EB, You LH, Wang N, Wang LT, Jin FY, et al. Downregulation of IncRNA TUG1 affects apoptosis and insulin secretion in mouse pancreatic beta cells. Cell Physiol Biochem. 2015;35(5):1892-904.

18. Zhang N, Geng T, Wang Z, Zhang R, Cao T, Camporez JP, et al. Elevated hepatic expression of $\mathrm{H} 19$ long noncoding RNA contributes to diabetic hyperglycemia. JCI Insight. 2018;3(10):e120304.

19. Zhao XY, Li S, DelProposto JL, Liu T, Mi L, Porsche C, et al. The long noncoding RNA BInc1 orchestrates homeostatic adipose tissue remodeling to preserve metabolic health. Mol Metab. 2018;14:60-70.
20. China Diabetes Association. China guideline for type 2 diabetes. Chin J Diabetes. 2018;10(1):4-67.

21. Livak KJ, Schmittgen TD. Analysis of relative gene expression data using real-time quantitative PCR and the 2(-Delta Delta C(T)) Method. Methods (San Diego, Calif). 2001;25(4):402-8.

22. Marchenko IV, Dubovyk YI, Tkach GF, Maksymova OS, Matlai OI, Ataman AV, et al. The association between enpp1 rs997509 polymorphism and type 2 diabetes mellitus development in Ukrainian population. Wiad Lek. 2018:71(3 pt 1):490-5.

23. Song L, Liu H, Wang Y, Wang Y, Liu J, Zhou Z, et al. Application of GC/ MS-based metabonomic profiling in studying the therapeutic effects of Huangbai-Zhimu herb-pair (HZ) extract on streptozotocin-induced type 2 diabetes in mice. J Chromatogr B Anal Technol Biomed Life Sci. 2015;997:96-104.

24. Wang Y, Hai B, Niu X, Ai L, Cao Y, Li R, et al. Chronic intermittent hypoxia disturbs insulin secretion and causes pancreatic injury via the MAPK signaling pathway. Biochem Cell Biol. 2017;95(3):415-20.

25. Narasimhan A, Chinnaiyan $M$, Karundevi B. Ferulic acid regulates hepatic GLUT2 gene expression in high fat and fructose-induced type-2 diabetic adult male rat. Eur J Pharmacol. 2015:761:391-7.

26. Rogoyski OM, Pueyo Jl, Couso JP, Newbury SF. Functions of long noncoding RNAs in human disease and their conservation in Drosophila development. Biochem Soc Trans. 2017;45(4):895-904.

27. Liu Y, Zhao M. InCaNet: pan-cancer co-expression network for human IncRNA and cancer genes. Bioinformatics (Oxford, England). 2016:32(10):1595-7.

28. Deng H, Wang JM, Li M, Tang R, Tang K, Su Y, et al. Long non-coding RNAs: new biomarkers for prognosis and diagnosis of colon cancer. Tumour Biol. 2017:39(6):1010428317706332.

29. Dong L, Lin W, Qi P, Xu MD, Wu X, Ni S, et al. Circulating long RNAs in serum extracellular vesicles: their characterization and potential application as biomarkers for diagnosis of colorectal cancer. Cancer Epidemiol Biomark Prev. 2016;25(7):1158-66.

30. Voight BF, Scott LJ, Steinthorsdottir V, Morris AP, Dina C, Welch RP, et al. Twelve type 2 diabetes susceptibility loci identified through large-scale association analysis. Nat Genet. 2010;42(7):579-89.

31. Pasmant E, Sabbagh A, Vidaud M, Bieche I. ANRIL, a long, noncoding RNA is an unexpected major hotspot in GWAS. FASEB J. 2011;25(2):444-8.

32. Imamura M, Maeda S, Yamauchi T, Hara K, Yasuda K, Morizono T, et al. A single-nucleotide polymorphism in ANK1 is associated with susceptibility to type 2 diabetes in Japanese populations. Hum Mol Genet. 2012:21(13):3042-9.

33. Willer CJ, Schmidt EM, Sengupta S, Peloso GM, Gustafsson S, Kanoni S, et al. Discovery and refinement of loci associated with lipid levels. Nat Genet. 2013;45(11):1274-83.

34. de Mello VD, Kolehmanien M, Schwab U, Pulkkinen L, Uusitupa M. Gene expression of peripheral blood mononuclear cells as a tool in dietary intervention studies: what do we know so far? Mol Nutr Food Res. 2012:56(7):1160-72

35. Diaz-Rua R, Palou A, Oliver P. Cpt1a gene expression in peripheral blood mononuclear cells as an early biomarker of diet-related metabolic alterations. Food Nutr Res. 2016;60:33554.

36. Mohamadi M, Ghaedi $H$, Kazerouni F, Erfanian Omidvar M, Kalbasi S, Shanaki M, et al. Deregulation of long noncoding RNA SNHG17 and TTC28-AS1 is associated with type 2 diabetes mellitus. Scand J Clin Lab Invest. 2019;79(7):519-23.

37. Erfanian Omidvar M, Ghaedi H, Kazerouni F, Kalbasi S, Shanaki M, Miraalamy $\mathrm{G}$, et al. Clinical significance of long noncoding RNA VIMAS1 and CTBP1-AS2 expression in type 2 diabetes. J Cell Biochem. 2019:120(6):9315-23.

38. Inoue T, Hagiyama M, Yoneshige A, Kato T, Enoki E, Maenishi O, et al. Increased ectodomain shedding of cell adhesion molecule 1 from pancreatic islets in type 2 diabetic pancreata: correlation with hemoglobin A1c levels. PLoS ONE. 2014;9(6):e100988.

39. Sharafshah A, Keshavarz P, Rezaei S, Farhadian N. Association and in silico studies of ENPP1 gene variants with type 2 diabetes mellitus in a Northern Iranian population. Gene. 2018;675:225-32.

40. Marchenko IV, Dubovyk YI, Matlai OI, Biesiedina AA, Kniazkova PV, Harbuzova YA. The analysis of association between ENPP1 K121Q polymorphism and risk factors of type 2 diabetes mellitus in Ukrainian population. Wiad Lek. 2018;71(4):815-20. 
41. Cirelli T, Nepomuceno R, Rios ACS, Orrico SRP, Cirelli JA, Theodoro LH, et al. Genetic polymorphisms in the Interleukins IL1B, IL4, and IL6 are associated with concomitant periodontitis and type 2 diabetes mellitus in Brazilian patients. J Periodontal Res. 2020;55(6):918-30.

42. Nuli R, Azhati J, Cai J, Kadeer A, Zhang B, Mohemaiti P. Metagenomics and faecal metabolomics integrative analysis towards the impaired glucose regulation and type 2 diabetes in Uyghur-related omics. J Diabetes Res. 2019;2019:2893041.

43. Schultze SM, Hemmings BA, Niessen M, Tschopp O. PI3K/AKT, MAPK and AMPK signalling: protein kinases in glucose homeostasis. Expert Rev Mol Med. 2012;14:e1.

44. Wang X, Chang X, Zhang P, Fan L, Zhou T, Sun K. Aberrant expression of long non-coding RNAs in newly diagnosed type 2 diabetes indicates potential roles in chronic inflammation and insulin resistance. Cell Physiol Biochem. 2017:43(6):2367-78.

45. Yan C, Li J, Feng S, Li Y, Tan L. Long noncoding RNA Gomafu upregulates Foxo1 expression to promote hepatic insulin resistance by sponging miR139-5p. Cell Death Dis. 2018;9(3):289.

46. Gao Y, Wu F, Zhou J, Yan L, Jurczak MJ, Lee HY, et al. The H19/let-7 doublenegative feedback loop contributes to glucose metabolism in muscle cells. Nucleic Acids Res. 2014;42(22):13799-811.

47. Ruan Y, Lin N, Ma Q, Chen R, Zhang Z, Wen W, et al. Circulating LncRNAs analysis in patients with type 2 diabetes reveals novel genes influencing glucose metabolism and islet beta-cell function. Cell Physiol Biochem. 2018;46(1):335-50.

48. Pan W, Sun W, Yang S, Zhuang H, Jiang H, Ju H, et al. LDL-C plays a causal role on T2DM: a Mendelian randomization analysis. Aging (Albany NY). 2020;12(3):2584-94.

49. Gress TW, Nieto FJ, Shahar E, Wofford MR, Brancati FL. Hypertension and antihypertensive therapy as risk factors for type 2 diabetes mellitus. Atherosclerosis Risk in Communities Study. N Engl J Med. 2000;342(13):905-12.

50. Kim MJ, Lim NK, Choi SJ, Park HY. Hypertension is an independent risk factor for type 2 diabetes: the Korean genome and epidemiology study. Hypertens Res. 2015;38(11):783-9.

51. Cabili MN, Trapnell C, Goff L, Koziol M, Tazon-Vega B, Regev A, et al. Integrative annotation of human large intergenic noncoding RNAs reveals global properties and specific subclasses. Genes Dev. 2011:25(18):1915-27.

\section{Publisher's Note}

Springer Nature remains neutral with regard to jurisdictional claims in published maps and institutional affiliations.
Ready to submit your research? Choose BMC and benefit from:

- fast, convenient online submission

- thorough peer review by experienced researchers in your field

- rapid publication on acceptance

- support for research data, including large and complex data types

- gold Open Access which fosters wider collaboration and increased citations

- maximum visibility for your research: over $100 \mathrm{M}$ website views per year

At BMC, research is always in progress.

Learn more biomedcentral.com/submissions 